\title{
Prevalence and Antimicrobial Resistances of Salmonella spp. Isolated from Wild Boars in Liguria Region, Italy
}

\author{
Elisabetta Razzuoli ${ }^{1, *(\mathbb{D})}$, Valeria Listorti ${ }^{1, *}$ (]) Isabella Martini ${ }^{1}$, Laura Migone ${ }^{1}$, Lucia Decastelli ${ }^{2}$, \\ Walter Mignone ${ }^{3}$, Enrica Berio ${ }^{3}$, Roberta Battistini ${ }^{4} \mathbb{D}$, Carlo Ercolini ${ }^{4}$, Laura Serracca ${ }^{4}$, Tiziana Andreoli ${ }^{5}$, \\ Monica Dellepiane ${ }^{5}$, Daniela Adriano ${ }^{2} \mathbb{D}$, Monica Pitti ${ }^{2}$, Daniela Meloni ${ }^{2}{ }^{\mathbb{D}}$ and Paola Modesto ${ }^{1, *} \mathbb{C}$
}

check for updates

Citation: Razzuoli, E.; Listorti, V.; Martini, I.; Migone, L.; Decastelli, L.; Mignone, W.; Berio, E.; Battistini, R.; Ercolini, C.; Serracca, L.; et al. Prevalence and Antimicrobial Resistances of Salmonella spp. Isolated from Wild Boars in Liguria Region, Italy. Pathogens 2021, 10, 568. https:/ / doi.org/10.3390/pathogens10050568

Academic Editor:

Marialaura Corrente

Received: 31 March 2021

Accepted: 5 May 2021

Published: 7 May 2021

Publisher's Note: MDPI stays neutral with regard to jurisdictional claims in published maps and institutional affiliations.

Copyright: (c) 2021 by the authors. Licensee MDPI, Basel, Switzerland. This article is an open access article distributed under the terms and conditions of the Creative Commons Attribution (CC BY) license (https:/ / creativecommons.org/licenses/by/ $4.0 /)$.
1 Department of Genoa, Istituto Zooprofilattico Sperimentale del Piemonte, Liguria e Valle d'Aosta, Piazza Borgo Pila 39/24, 16129 Genoa, Italy; isabella.martini@izsto.it (I.M.); laura.migone@izsto.it (L.M.)

2 Department of Turin, Istituto Zooprofilattico Sperimentale del Piemonte, Liguria e Valle d'Aosta, Via Bologna 148, 10154 Turin, Italy; lucia.decastelli@izsto.it (L.D.); daniela.adriano@izsto.it (D.A.); monica.pitti@izsto.it (M.P.); daniela.meloni@izsto.it (D.M.)

3 Department of Imperia, Istituto Zooprofilattico Sperimentale del Piemonte, Liguria e Valle d'Aosta, Via Nizza 4, 18100 Imperia, Italy; walter.mignone@izsto.it (W.M.); enrica.berio@izsto.it (E.B.)

4 Department of La Spezia, Istituto Zooprofilattico Sperimentale del Piemonte, Liguria e Valle d'Aosta, Via degli Stagnoni 96, 19100 La Spezia, Italy; roberta.battistini@izsto.it (R.B.); carlo.ercolini@izsto.it (C.E.); laura.serracca@izsto.it (L.S.)

5 Department of Savona, Istituto Zooprofilattico Sperimentale del Piemonte, Liguria e Valle d'Aosta, Via Martiri 6, 17056 Savona, Italy; tiziana.andreoli@izsto.it (T.A.); monica.dellepiane@izsto.it (M.D.)

* Correspondence: elisabetta.razzuoli@izsto.it (E.R.); valeria.listorti@izsto.it (V.L.); paola.modesto@izsto.it (P.M.); Tel.: +39-010-542274 (E.R., V.L. \& P.M.); Fax: +39-010-566654 (E.R., V.L. \& P.M.)

Abstract: Salmonella spp. is an important zoonotic agent. Wild boars might host this pathogen in the intestinal tract and might represent a risk for Salmonella spp. transmission to humans. Wild boars are widely spread in Liguria, due to the environmental characteristics of the region. The aim of the study was the isolation, typing, and investigation of antimicrobial susceptibility of the isolated strains of Salmonella spp. During the 2013-2017 hunting seasons, 4335 livers of wild boars were collected and analyzed for the presence of Salmonella spp. A total of 260 strains of Salmonella spp. were isolated and characterized, with a prevalence of $6 \%$. The isolated strains belonged to all six Salmonella enterica subspecies. Most of them were identified as Salmonella enterica subs. enterica of which 31 different serotypes were identified. The dominating serotype identified was $S$. Enteritidis. The antimicrobial resistance profiles of the isolated strains were analyzed against sixteen molecules. Of the isolated strains, $94.6 \%$ were resistant to at least one of the tested antimicrobials. This study showed the circulation of resistant Salmonella spp. strains in the wild boar population living in this area of Italy, underling the potential risk for these animals to disseminate this pathogen and its antimicrobial resistances.

Keywords: Salmonella spp.; wild boars; antimicrobic resistance

\section{Introduction}

Salmonella spp. is one of the most important causes of human zoonosis in Europe [1]. Many serotypes have been isolated from the intestinal tract of a wide range of animals, including wild boars [2], which may act as a reservoir and disseminate pathogenic strains of Salmonella spp. [3]. In Europe, the twenty most frequent serotypes of Salmonella spp. causing illness in humans as reported by the European Authority for the Food Safety (EFSA), belong to the S.enterica subs. enterica, and among them the three most common are $S$. Enteritidis, S. Typhimurium, and $S$. Typhimurium monophasic variant 1,4,[5],12:i- [1]. Antimicrobial resistance is one of the most challenging health problems worldwide [4]. Wild boars are an important reservoir of antimicrobial-resistant bacteria and could be used as a sentinel species for surveillance [5]. Antimicrobial-resistant pathogens, including Salmonella 
spp., have been widely isolated from this species [6-8]. Wild boars are omnivorous and considering their behavior and habit of rooting through waste in urban areas, sources of exposure to Salmonella spp. include the consumption of contaminated carcasses and contact with infected farmed animals [9] or other wild animals. These animals could represent a risk for the transmission of Salmonella spp. during evisceration, through consumption of meat and meat products [2], from contamination of vegetables in agricultural areas, and through direct or indirect contact with farmed animals.

Wild boars are widely present in Italy and in Europe with an increasing prevalence $[10,11]$. In particular, in the area of the survey, the Liguria region, the characteristics of the territory support a wide occurrence of this species as well as in urban and periurban areas. In the Liguria region, the presence of wild boars has been recognized as a risk for zoonoses transmission. It has been observed that two important zoonotic agents, Leptospira spp. [12] and Yersinia spp., circulate in wild boar populations living in this area, and for the second pathogen, the presence of multidrug-resistant strains has been recently observed [13]. The aim of the study was to investigate the presence of Salmonella spp. in the liver of wild boars hunted in Liguria region and to analyze the profiles of antibiotic resistance of the isolated strains. This survey was conducted during regional wild animal monitoring plans, from 2013 to 2017. The monitoring plans were carried out in order to investigate the presence of infectious diseases representing a risk for public health in wild boars and other wild animals.

\section{Results}

\subsection{Salmonella spp. Typing}

Of the 4335 samples analyzed, 540 were positive to PCR screening. The positive samples where then cultured and among them a total of 260 Salmonella spp. strains were isolated and typed (Table 1 ), with a prevalence of $6 \%$. Most of the isolated strains $(157 / 260,60.4 \%)$ were typed as S. enterica subs. enterica of which a total of 31 serotypes were identified (Table 1). Among them, seven of the twenty most frequent Salmonella spp. serotypes responsible for illness in humans in Europe were identified: $S$. Enteritidis (20/260, 7.7\%), S. Typhimurium (10/260,3.8\%), S. Typhimurium monophasic variant 1,4,[5],12:i:- (4/260, 1.5\%), S. Infantis (3/260, 1.2\%), S. Newport (8/260, 3.1\%), S. Napoli $(21 / 260,8 \%)$, and $S$. Coeln $(9 / 260,3.5 \%)$. No clinical signs were reported by hunters and Salmonella spp. isolation was not associated with the presence of macroscopic lesions referable to salmonellosis.

Table 1. Subspecies of S. enterica and serotypes of Salmonella enterica subs. enterica isolated.

\begin{tabular}{ccc}
\hline Isolated Serotypes or Subspecies & Number/260 & Percentage/Tot. \\
\hline S. enterica subs. enterica (not serotyped) & 5 & $1.9 \%$ \\
\hline S. Enteritidis & 20 & $7.7 \%$ \\
\hline S. Typhimurium & 10 & $3.8 \%$ \\
\hline S. Typhiumurium monophasic variant 1,4,[5],12:i:- & 4 & $1.5 \%$ \\
\hline S. Infantis & 3 & $1.2 \%$ \\
\hline S. Newport & 8 & $3.1 \%$ \\
\hline S. Napoli & 21 & $8 \%$ \\
\hline S. Coeln & 9 & $3.5 \%$ \\
\hline S. Brandenburg & 3 & $1.2 \%$ \\
\hline S. Veneziana & 10 & $3.8 \%$ \\
\hline S. Thompson & 8 & $3.1 \%$ \\
\hline
\end{tabular}


Table 1. Cont.

\begin{tabular}{|c|c|c|}
\hline Isolated Serotypes or Subspecies & Number/260 & Percentage/Tot. \\
\hline S. Canada & 8 & $3.1 \%$ \\
\hline S. Oxford & 7 & $2.7 \%$ \\
\hline S. Muenster & 6 & $2.3 \%$ \\
\hline S. Kottbus & 5 & $1.9 \%$ \\
\hline S. Galil & 4 & $1.5 \%$ \\
\hline S. Kimuenza & 6 & $2.3 \%$ \\
\hline S. Banjul & 4 & $1.5 \%$ \\
\hline S. Stourbridge & 3 & $1.2 \%$ \\
\hline S. Juba & 2 & $0.8 \%$ \\
\hline S. Arechavaleta & 2 & $0.8 \%$ \\
\hline S. Atakpame & 1 & $0.4 \%$ \\
\hline S. Stoneferry & 1 & $0.4 \%$ \\
\hline S. Umbilo & 1 & $0.4 \%$ \\
\hline S. Goldocoast & 1 & $0.4 \%$ \\
\hline S. Grampiam & 1 & $0.4 \%$ \\
\hline S. Ablogame & 1 & $0.4 \%$ \\
\hline S. Massakory & 1 & $0.4 \%$ \\
\hline S. Bispebjerg & 1 & $0.4 \%$ \\
\hline S. Bahrenfeld & 1 & $0.4 \%$ \\
\hline S. enterica subs. salamae & 53 & $20.4 \%$ \\
\hline S. enterica subs. arizonae & 13 & $5 \%$ \\
\hline S. enterica subs. diarizonae & 29 & $11.2 \%$ \\
\hline S. enterica subs. houtenae & 7 & $2.7 \%$ \\
\hline S. enterica subs. indica & 1 & $0.4 \%$ \\
\hline
\end{tabular}

\subsection{Antimicrobial Susceptibility Analysis}

The strains were classified as susceptible, intermediate, or resistant. The details of the antimicrobial susceptibility of the isolated strains are reported in Table S1 in the Supplementary Material. The summary concerning the antimicrobial susceptibility of the analyzed strains is reported in Table 2 . A total of $94.6 \%$ of the analyzed strains $(246 / 260)$ were resistant to at least one of the tested molecules; $40 \%(98 / 260)$ to two or more; $17.3 \%$ $(45 / 260)$ to three or more; and 9.6\% (25/260) of the isolated strains resulted resistant to four of more antimicrobials. In particular, the higher resistances were observed in two strains of $S$. Typhimurium monophasic variant 1,4,[5],12:i:-, three strains of $S$. Brandenburg, and one strain of $S$. enterica subs. salamae that resulted resistant to seven antimicrobials (Table 3). Most of the resistances were observed against sulfadiazine + sulfamerazine + sulfamethazine; in fact $96 \%$ of the strains tested resulted resistant to these molecules. Less than the $1 \%$ of tested strains resulted resistant to chloramphenicol, colistin, ceftazidime, enrofloxacin, and nalidixic acid. No one strain resulted resistant to ciprofloxacin. The majority of the intermediate susceptibilities were observed against kanamycin (43\%), streptomycin $(30.2 \%)$, and tetracycline $(23.4 \%)$ 
Table 2. Percentage of isolated strain which resulted susceptible, intermediate, or resistant to the tested antimicrobials.

\begin{tabular}{cccc}
\hline Antibiotic & Susceptible & Intermediate & Resistant \\
\hline Ampicillin & $206 / 260(79.2 \%)$ & $29 / 260(11.2 \%)$ & $25 / 260(9.6 \%)$ \\
\hline Amoxicillin + Clavulanic Acid & $171 / 217(78.8 \%)$ & $33 / 217(15.2 \%)$ & $13 / 217(6 \%)$ \\
\hline Chloramphenicol & $247 / 251(98.4 \%)$ & $3 / 251(1.2 \%)$ & $1 / 251(0.4 \%)$ \\
\hline Cefalotin & $197 / 260(75.8 \%)$ & $47 / 260(18 \%)$ & $16 / 260(6.2 \%)$ \\
\hline Cefotaxime & $235 / 258(91 \%)$ & $20 / 258(7.8 \%)$ & $3 / 258(1.2 \%)$ \\
\hline Ciprofloxacin & $258 / 260(99.2 \%)$ & $2 / 260(0.8 \%)$ & $0 / 260(0 \%)$ \\
\hline Colistin & $237 / 245(96.7 \%)$ & $7 / 245(2.9 \%)$ & $1 / 245(0.4 \%)$ \\
\hline Ceftazidime & $195 / 249(78.3 \%)$ & $52 / 249(20.9 \%)$ & $2 / 249(0.8 \%)$ \\
\hline Enrofloxacin & $190 / 207(91.8 \%)$ & $16 / 207(7.7 \%)$ & $1 / 207(0.5 \%)$ \\
\hline Gentamicin & $223 / 257(86.8 \%)$ & $27 / 257(10.5 \%)$ & $7 / 257(2.7 \%)$ \\
\hline Kanamycin & $118 / 230(51.3 \%)$ & $99 / 230(43 \%)$ & $13 / 230(5.7 \%)$ \\
\hline Nalidixic acid & $219 / 253(86.6 \%)$ & $32 / 253(12.6 \%)$ & $2 / 253(0.8 \%)$ \\
\hline Streptomycin & $106 / 258(41 \%)$ & $78 / 258(30.2 \%)$ & $28 / 258(10.8 \%)$ \\
\hline Sulfadiazine + Sulfamerazine + Sulfamethazine & $6 / 248(2.4 \%)$ & $4 / 248(1.6 \%)$ & $238 / 248(96 \%)$ \\
\hline Sulfamethoxazole + Trimethoprim & $153 / 260(58.8 \%)$ & $50 / 260(19.2 \%)$ & $57 / 260(21.9 \%)$ \\
\hline Tetracycline & $147 / 260(56.5 \%)$ & $61 / 260(23.4 \%)$ & $52 / 260(20 \%)$ \\
\hline
\end{tabular}

The percentages of strains determined to be antimicrobial resistant, belonging to the most frequent serotypes of Salmonella spp. causing human illness in Europe, are reported in Table 4 . The strains of $S$. Typhimurium monophasic variant 1,4,[5],12:i:- showed the most noteworthy antimicrobial resistances. In fact, $100 \%$ of the analyzed strains showed resistance to: ampicillin, amoxicillin-clavulanic acid, streptomycin, trimethoprimsulfamethoxazole, and tetracycline; $75 \%$ of the analyzed strains showed resistance to cefalotin. The strains of $S$. Coeln showed resistance to nine molecules but in a lower rate. In particular, $100 \%$ of the analyzed strains showed resistance to sulfadiazine + sulfamerazine + sulfamethazine; $67 \%$ to tetracycline; and $22 \%$ to amoxicillin-clavulanic acid, streptomycin, and trimethoprim-sulfamethoxazole. A total of $11 \%$ of the analyzed strains resulted resistant to ampicillin, cefalotin, cefotaxime, and ceftazidime. The $S$. Napoli isolated strains showed resistance to four molecules. In particular, $86 \%$ of the isolated strains resulted resistant to sulfadiazine + sulfamerazine + sulfamethazine; $19 \%$ to ampicillin; $10 \%$ to tetracycline; and 5\% to streptomycin. The $S$. Typhimurium isolated strains resulted resistant to four molecules. A total of $100 \%$ of the analyzed strains resulted resistant to sulfadiazine + sulfamerazine + sulfamethazine; $30 \%$ to trimethoprim-sulfamethoxazole; $11 \%$ to amoxicillin-clavulanic acid; and $10 \%$ to ampicillin. The $S$. Newport isolated strains resulted resistant to three molecules. Among them, $100 \%$ of the strains resulted resistant to sulfadiazine + sulfamerazine + sulfamethazine; $63 \%$ to streptomycin; and $13 \%$ to trimethoprim-sulfamethoxazole. The $S$. Infantis isolated strains resulted resistant to three of the tested molecules. In particular, $100 \%$ of the strains resulted resistant to sulfadiazine + sulfamerazine + sulfamethazine and $33 \%$ to cefalotin and tetracycline. Also, the $S$. Enteritidis isolated strains resulted resistant to three molecules: $100 \%$ was resistant to sulfadiazine + sulfamerazine + sulfamethazine; $25 \%$ was resistant to trimethoprim-sulfamethoxazole; and $5 \%$ was resistant to tetracycline. Considering these strains in total, the higher intermediate susceptibilities were observed against kanamycin $(22 \%)$, tetracycline $(16.7 \%)$, and streptomycin $(9.1 \%)$. 
Table 3. Antimicrobial resistance profile of multidrug-resistant strains (to 4 or more antimicrobials). In bold: antimicrobials considered "Highest Priority Critically Important".

\begin{tabular}{|c|c|c|}
\hline Year & Strain & Antimicrobial Resistances \\
\hline 2014 & S. Typhimurium monophasic variant $1,4,[5], 12: i:-$ & A-AMC-KF-S-SSS-SXT-T \\
\hline 2014 & S. Typhimurium monophasic variant $1,4,[5], 12: i:-$ & A-AMC-KF-S-SSS-SXT-T \\
\hline 2014 & S. Typhimurium monophasic variant $1,4,[5], 12: \mathrm{i}:-$ & A-AMC-KF-S-SXT-T \\
\hline 2014 & S. Typhimurium monophasic variant $1,4,[5], 12: i:-$ & A-AMC-S-SSS-SXT-T \\
\hline 2013 & S. Napoli & A-S-SSS-T \\
\hline 2013 & S. Coeln & KF-CST-CAZ-S-SSS-T \\
\hline 2015 & S. Coeln & S-SSS-SXT-T \\
\hline 2013 & S. Brandenburg & A-AMC-KF-CTX-K-SSS-T \\
\hline 2013 & S. Brandenburg & A-AMC-KF-CTX-K-SSS-T \\
\hline 2013 & S. Brandenburg & A-AMC-KF-CTX-K-SSS-T \\
\hline 2013 & S. Stourbridge & S-SSS-SXT-T \\
\hline 2013 & S. Stourbridge & S-SSS-SXT-T \\
\hline 2013 & S. Muenster & A-AMC-KF-SSS \\
\hline 2013 & S. Atakpame & A-AMC-KF-SSS-SXT-T \\
\hline 2016 & S. Veneziana & G-K-S-SSS-SXT-T \\
\hline 2016 & S. Veneziana & G-K-SSS-SXT-T \\
\hline 2016 & S. Canada & A-SSS-SXT-T \\
\hline 2016 & S. Bahrenfeld & G-K-SSS-SXT-T \\
\hline 2013 & S. enterica subs. salamae & A-AMC-C-KF-SSS-SXT-T \\
\hline 2014 & S. enterica subs. salamae & A-KF-SSS-T \\
\hline 2016 & S. enterica subs. salamae & A-KF-S-SSS-SXT-T \\
\hline 2016 & S. enterica subs. salamae & G-K-S-SSS \\
\hline 2016 & S. enterica subs. salamae & ENR-G-K-SSS-SXT \\
\hline 2016 & S. enterica subs. salamae & G-K-SSS-SXT-T \\
\hline 2014 & S. enterica subs. diarizonae & S-SSS-SXT-T \\
\hline
\end{tabular}

A: ampicillin, AMC: amoxicillin/clavulanic acid 2:1, C: chloramphenicol, KF: cefalotin, CTX: cefotaxime, CST: colistin, CAZ: ceftazidime, ENR: enrofloxacin, G: gentamicin, K: kanamycin, S: streptomycin, SSS: Sulfadiazine + Sulfamerazine + Sulfamethazine, SXT: trimethoprim-sulfamethoxazole, T: tetracycline. 


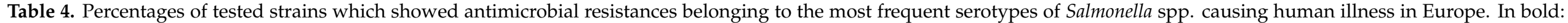
antimicrobials considered "Highest Priority Critically Important".

\begin{tabular}{|c|c|c|c|c|c|c|c|c|c|c|c|c|c|c|c|c|}
\hline \multirow{2}{*}{ Serotype } & \multicolumn{16}{|c|}{ Antimicrobials } \\
\hline & $\mathrm{A}$ & AMC & $\mathrm{C}$ & KF & CTX & CIP & CST & CAZ & ENR & G & K & NAL & $S$ & SSS & SXT & $\mathrm{T}$ \\
\hline S. Enteritidis & $-*$ & - & - & - & - & - & - & - & - & - & - & - & - & $\begin{array}{l}20 / 20 \\
(100 \%)\end{array}$ & $\begin{array}{l}5 / 20 \\
(25 \%)\end{array}$ & $\begin{array}{l}1 / 20 \\
(5 \%)\end{array}$ \\
\hline S. Typhimurium & $\begin{array}{c}1 / 10 \\
(10 \%)\end{array}$ & $\begin{array}{c}1 / 9 \\
(11 \%)\end{array}$ & - & - & - & - & - & - & - & - & - & - & - & $\begin{array}{c}6 / 6 \\
(100 \%)\end{array}$ & $\begin{array}{l}3 / 10 \\
(30 \%)\end{array}$ & - \\
\hline $\begin{array}{l}\text { S. Typhimurium } \\
\text { monoph. variant }\end{array}$ & $\begin{array}{c}4 / 4 \\
(100 \%)\end{array}$ & $\begin{array}{c}4 / 4 \\
(100 \%)\end{array}$ & - & $\begin{array}{c}3 / 4 \\
(75 \%)\end{array}$ & - & - & - & - & - & - & - & - & $\begin{array}{c}4 / 4 \\
(100 \%)\end{array}$ & $\begin{array}{c}3 / 3 \\
(100 \%)\end{array}$ & $\begin{array}{c}4 / 4 \\
(100 \%)\end{array}$ & $\begin{array}{c}4 / 4 \\
(100 \%)\end{array}$ \\
\hline S. Infantis & - & - & - & $\begin{array}{c}1 / 3 \\
(33 \%)\end{array}$ & - & - & - & - & - & - & - & - & - & $\begin{array}{c}3 / 3 \\
(100 \%)\end{array}$ & - & $\begin{array}{c}1 / 3 \\
(33 \%)\end{array}$ \\
\hline S. Newport & - & - & - & & - & - & - & - & - & - & - & - & $\begin{array}{c}5 / 8 \\
(63 \%)\end{array}$ & $\begin{array}{c}8 / 8 \\
(100 \%)\end{array}$ & $\begin{array}{c}1 / 8 \\
(13 \%)\end{array}$ & - \\
\hline S. Napoli & $\begin{array}{c}4 / 21 \\
(19 \%)\end{array}$ & - & - & - & - & - & - & - & - & - & - & - & $\begin{array}{l}1 / 21 \\
(5 \%)\end{array}$ & $\begin{array}{l}18 / 21 \\
(86 \%)\end{array}$ & - & $\begin{array}{l}2 / 21 \\
(10 \%)\end{array}$ \\
\hline S. Coeln & $\begin{array}{c}1 / 9 \\
(11 \%)\end{array}$ & $\begin{array}{c}2 / 9 \\
(22 \%)\end{array}$ & - & $\begin{array}{c}1 / 9 \\
(11 \%)\end{array}$ & - & - & $\begin{array}{c}1 / 9 \\
(11 \%)\end{array}$ & $\begin{array}{c}1 / 9 \\
(11 \%)\end{array}$ & - & - & - & - & $\begin{array}{c}2 / 9 \\
(22 \%)\end{array}$ & $\begin{array}{c}9 / 9 \\
(100 \%)\end{array}$ & $\begin{array}{c}2 / 9 \\
(22 \%)\end{array}$ & $\begin{array}{c}6 / 9 \\
(67 \%)\end{array}$ \\
\hline
\end{tabular}

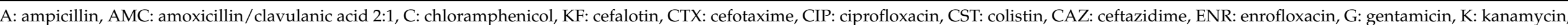
NAL: nalidixic acid, S: streptomycin, SSS: Sulfadiazine + Sulfamerazine + Sulfamethazine, SXT: trimethoprim-sulfamethoxazole, T: tetracycline. - *: Resistance not observed. 


\section{Discussion}

Salmonella spp. is the second cause of human zoonosis in Europe. Many serotypes can be involved in a morbid event causing different clinical signs. The results obtained in our study confirm the possible role of wild boars in the transmission of Salmonella spp.

Indeed, the presence of Salmonella spp. in the deep part of muscles of wild boars hunted for consumption in Italy was already demonstrated [14] and it was also reported on the carcasses and meat cuts of wild boars in European countries [15]. The presence of Salmonella spp. in the liver, that is usually eaten lightly cooked or used for cured meat preparation, suggests the existence of a risk of transmission of Salmonella spp. to humans. To our knowledge, no salmonellosis cases following consumption of meat from wild boars have been reported in Italy. This is possibly due to the low prevalence of this pathogen, which was reported in studies conducted in Italy using different matrices (i.e., faeces or intestinal content) [6-8]. Another reason could be the low amount of wild animal meat consumed compared with the total amount of meat consumed. In addition, the EFSA reports do not specify the origin of the meat causing human salmonellosis in European countries [1]. However, during an outbreak of $S$. Cholerasuis var. Kusendorf in wild boars in Italy, the same serotype was isolated in a human case of salmonellosis. The phylogenetic and PFGE analyses of the isolated strains suggested a high degree of similarity between the human isolates and the isolates from the wild boar outbreak. This study suggested the potential role of wild boars in spreading $S$. Cholerasuis to humans [16]. However, Salmonella spp. can be released from infected wild boars into the environment, causing contamination of surface water, direct or indirect contamination of crops, and could be transmitted to farmed animals. The risk of direct or indirect transmission of Salmonella spp. from wild boars to humans is considered possible as previously reported by other authors [2]. Recent studies demonstrated the different ability of Salmonella strains to modulate innate immunity; in particular, it was demonstrated that IL-8 expression is very important for Salmonella spp. invasion of enterocytes [17,18]. In this respect, Razzuoli and coworkers demonstrated that different strains of $S$. diarizonae isolated in wild boars can have different pathogenicity [18]. Wild boars may act as healthy carriers for Salmonella spp. [3], but stressor conditions like the presence of concomitant viral diseases [19] or pollutants such as cadmium [20] could determine a high susceptibility to Salmonella spp., and clinical outbreaks could occur [21]. The $6 \%$ prevalence of Salmonella spp. observed in this study is similar to those reported in previous studies conducted in central Italy $[6,8]$ and northwestern Italy [7], where a prevalence of $4.8 \%, 7.2 \%$, and $10.8 \%$ were reported. S. enterica subs. enterica is the most common subspecies isolated in our study. This observation is consistent with other studies conducted in wild boars $[3,6,22]$. The main location of Salmonella spp. in non-adapted hosts is the intestinal tract. The use of liver samples for this study could have led us to underestimate the prevalence of Salmonella spp, but the liver was chosen for two reasons. The study was mainly conducted for food hygiene purposes and the liver is an edible part traditionally consumed in this part of Italy and also used for the production of cured meats; therefore, it could represent a risk for the transmission of Salmonella spp. to humans. The second reason was a practical trade-off. Sampling was carried out during the evisceration process by the hunters and the other parts of the carcasses were left at the disposal of hunters to then be consumed. In practice, it was better to not use the intestinal content because its collection could have represented a risk of contamination of the carcasses and would not have guaranteed transport in safe hygienic conditions to the laboratory. Other studies have investigated the presence of Salmonella spp. in this organ [8].

The other subspecies of Salmonella spp. represent $40 \%$ of the isolated strains. Among them, S. enterica subs. salamae is the most frequently isolated subspecies $(20.4 \%)$. This subspecies of Salmonella has also been isolated in a high rate in an investigation previously conducted in Italy in wild boars [6]. Among the strains most frequently implicated in human illness in Europe [1], our results showed the presence of $S$. Enteritidis, $S$. Typhimurium, S. Typhimurium monophasic variant 1,4,[5],12:i:-, $S$. Infantis, $S$. Newport, $S$. Napoli, and $S$. 
Coeln. These strains were previously reported in wild boars in Italy [3,6-8]. The relative high number $(21 / 260,8 \%)$ of isolates belonging to $S$. Napoli serotype is interesting; in fact it is a re-emerging serotype in Italy. It has been hypothesized that the environment can act as reservoir for this serotype from where it can spill over to humans and animals [23]. This serotype has been already isolated form wild boars in Italy [3,24]. Our investigation confirms the circulation of $S$. Napoli in wild boars that can represent a route of dissemination of this serotype in humans and farmed animals, directly or contaminating surface water. Another interesting finding is the isolation of S. Stourbridge, a rare serotype that was involved in some severe outbreaks in Germany in 2016. Human patients presented severe clinical signs and two had a fatal outcome. Unfortunately, the source of infection has not been identified [25]. Following these outbreaks, ECDC asked for collaboration and sharing of data about the identification of $S$. Stourbridge from non-human sources. Indeed, few literature data are available about $S$. Stourbridge isolation, mainly from human patients and cattle [26]. To the extent of our knowledge, this is the first description of $S$. Stourbridge from wild boars in Italy, while it has already been described from a wild boar in Switzerland [27]. The present result suggests that wild boar are probably responsible for the $S$. Stourbridge source of infection. $S$. Cholerasuis is a host-adapted serotype, an agent of swine paratyphoid [28], and in our study, we did not isolate this serotype of Salmonella. The hunted wild boars did not show clinical signs and the isolation of Salmonella spp. were not associated with the presence of macroscopic lesions. The presence of $S$. Cholerasuis has been mainly reported in domestic pigs [29] and a possible mutual exposure to this pathogen between domestic pigs and wild boars has been hypostatised [30]. In our study, this serotype was not found.

The Liguria region has a very low density of farmed pigs with few animals at each farm site. This led us to hypothesize that contact between farmed pigs and wild boars is improbable, indicating a low probability of spread of this serotype in the wild boar population, despite a limited exchange of Salmonella spp. having been observed among domestic pigs and wild boars in Italy [31]. Moreover, the presence of this pathogen, to the extent of our knowledge, was not reported for farmed pigs in this area of Italy, and studies conducted in adjoining regions in wild boars did not detect this serotype too [8]. Wild animals might act as reservoir for antimicrobial resistance pathogens. It has been suggested that wildlife living in urban areas show higher antimicrobial resistances than animals living in remote areas [32], due to the possibility of contact with resistant bacteria and selective agents. Wild boars have contact with humanized environments, thus is a species that could be implicated in the cycle of antimicrobial resistance transmission [5]. In our study we confirm this evidence, showing that almost $94.6 \%$ of the isolated Salmonella spp. strains are characterized by a resistance to at least one antimicrobial tested. The highest resistances were observed against sulfonamides. In particular, $96 \%$ of the tested strains resulted resistant to the combination of sulfadiazine + sulfamerazine + sulfamethazine (triple-sulfa) and $21.9 \%$ to the combination of sulfamethoxazole + trimethoprim. Our results confirm the well-known, widespread antimicrobial resistance against these molecules, which, for this reason, have been less used by the clinicians for a long time [33]. A total of $20 \%$ of the tested strains resulted resistant to tetracycline; the antimicrobial resistance is well established for this molecule, and the use is limited only in infection with confirmed susceptibility [34]. A total of $10.9 \%$ of the isolated strains resulted resistant to streptomycin. Our results are consistent with other studies on the presence of Salmonella spp. in wild mammals and in other territories, in which the majority of the resistances reported are against the same classes of antimicrobials $[6,7,22,35]$ and in contrast with the results of a recent study conducted in central Italy in wild boars [8] where no resistances against tetracycline were observed, and a higher rate of antimicrobial resistance to streptomycin was reported. In our study, the antimicrobial susceptibility against molecules considered "Highest Priority Critically Important" antimicrobials for human medicine has been tested, among them, quinolones (nalidixic acid and ciprofloxacin) and cephalosporines of third generation (cefotaxime, ceftazidime). Both of these classes of antimicrobials are known to 
select for resistant Salmonella spp. and E. coli strains in animals, and at the same time, are among the few available therapies for serious Salmonella spp. and E. coli infections [36]. In our study, we also tested the antimicrobial susceptibility against colistin. This molecule belongs to the polymixines, which are known to select for plasmid-mediated polymyxinresistant $E$. coli in food animals, but at the same time, are one of the few available therapies for serious Enterobactericeae and Pseudomonas aeruginosa multi-resistant infections [36]. Fortunately, most of the isolated strains are sensitive to these antimicrobials. Considering the quinolones, only $0.8 \%$ of the tested strains resulted resistant to nalidixic acid, and no one strain resulted resistant to ciprofloxacin. The analysis of the antimicrobial susceptibility against cephalosporin of third generation also revealed a low rate of antimicrobial resistance. In particular, $1.2 \%$ of the isolated strains resulted resistant to cefotaxime and $0.8 \%$ to ceftazidime. Only $0.4 \%$ of the isolated strains resulted resistant to colistin.

The observed antimicrobial resistance to these molecules is lower than that reported in other studies conducted in wild boars; however, these studies considered a lower number of Salmonella spp. strains $[6,8,21]$. The majority of the intermediate susceptibility was observed against kanamycin (43\%), streptomycin $(30.2 \%)$, and tetracycline $(23.4 \%)$. However, considering only the seven serotypes, among the most frequently isolated in human illness in Europe, the percentage of intermediate susceptibility are lower when compared to all tested strains: kanamycin $(22 \%)$, streptomycin $(9.1 \%)$, and tetracycline $(16.7 \%)$. Considering all the tested strains, the highest intermediate susceptibility against the "Highest Priority Critically Important" antimicrobials was observed against ceftazidime (20.9\%), followed by nalidixic acid $(12.6 \%)$, cefotaxime $(7.8 \%)$, colistin $(2.9 \%)$, and ciprofloxacin $(0.8 \%)$. As in the previous case, considering the strains of the seven serotypes, among the most frequently isolated in human illness in Europe, the percentage of intermediate strains was lower: ceftazidime $(7.6 \%)$, cefotaxime $(3 \%)$, followed by nalidixic acid $(2.3 \%)$, and colistin $(0.8 \%)$; no intermediate susceptibility was observed against ciprofloxacin. Considering these observations in total, we can assert that, despite the presence of multi-drug resistances, the isolated strains present a high sensitivity rate against "Highest Priority Critically Important" antimicrobials in use.

\section{Materials and Methods}

\subsection{Samples Collection}

The survey was conducted during the hunting seasons from 2013-2017 (October to January). During the evisceration of hunted wild boars, 4335 livers were collected and analyzed for the presence of Salmonella spp. The area of the sampling was the Liguria region, in the northwest of Italy. The liver of each wild boar was collected by the hunters using dedicated knives and sterile bags provided by the laboratory and within $4 \mathrm{~h}$ after collection were transported refrigerated to the laboratory. The hunters were trained by laboratory workers on the procedures for the evisceration.

\subsection{Salmonella Isolation and Typing}

Twenty-five grams of each liver were sampled in the deep part of the organ, enriched (1:10) in buffered peptone water (BPW), homogenized in a Stomacher blender, and incubated for $18 \mathrm{~h}$ at $37 \pm 1{ }^{\circ} \mathrm{C}$. PCR method was used for screening analysis. The DNA was extracted and amplified using the iQ-Check Salmonella II PCR Detection kit (Bio-rad, Milan, Italy), according to the instructions of the producer. The isolation of Salmonella strains from PCR-positive samples was made according to ISO 6579:2002/COR. 1, 2004 (Microbiology of food and animal feeding stuffs: horizontal method for the detection of Salmonella spp.). Briefly, after the pre-enrichment, $100 \mu \mathrm{L}$ of sample was added to $10 \mathrm{~mL}$ of Rappaport Vassiliadis modified broth (RVS), and $1 \mathrm{~mL}$ of sample was added to $10 \mathrm{~mL}$ of Muller-Kauffmann tetrathionate-novobiocin broth (MKTTn), respectively. RVS broth was incubated for $24 \mathrm{~h}$ at $41.5 \pm 1{ }^{\circ} \mathrm{C}$ and the MKTTn broth for $24 \mathrm{~h}$ at $37 \pm 1{ }^{\circ} \mathrm{C}$. After the incubation, the samples were distributed onto plates of selective media, brilliant green agar (BGA) and xylose lysine deoxycholate agar (XLD). The plates were incubated for $24 \mathrm{~h}$ 
at $37 \pm 1{ }^{\circ} \mathrm{C}$, and then suspected colonies were transferred on nutrient agar and incubated at $37 \pm 1{ }^{\circ} \mathrm{C}$ for $20-24 \mathrm{~h}$. After this step, the suspected colonies were seeded in triple sugar iron (TSI) to evaluate $\mathrm{H}_{2} \mathrm{~S}$ production and glucose, lactose, and sucrose fermentation. The presence of Salmonella was confirmed using appropriate biochemical miniaturized tests (API 20E ${ }^{\circledR}$, Biomerieux, Lyon, France) and serological tests. Serotype identification of the isolated strains was carried out according to ISO/TR 6579-3, 2014 (Microbiology of the food chain-Horizontal method for the detection, enumeration and serotyping of Salmonella-Part 3: Guidelines for serotyping of Salmonella spp.).

\subsection{Antimicrobial Susceptibility Analysis}

The Kirby-Bauer disk diffusion test was performed according to Clinical and Laboratory Standard Institute (CLSI) guidelines [37] using Mueller-Hinton agar plates (Microbiol, Uta, Italy) and following the indications of the Italian National Reference Laboratory. The antimicrobials and concentrations $(\mu \mathrm{g})$ used were: ampicillin $(\mathrm{A}, 10)$, amoxicillin/clavulanic acid 2:1 (AMC, 30), chloramphenicol (C, 30), cefalotin (KF, 30), cefotaxime (CTX, 5), cyprofloxacin (CIP, 5), colistin (CST, 10), ceftazidime (CAZ, 10), enrofloxacin (ENR, 5), gentamicin $(G, 10)$, kanamycin $(K, 30)$, nalidixic acid (NAL, 30), streptomycin $(S$, 10), triple-sulfa (SSS, 250), trimethoprim-sulfamethoxazole (SXT, 1.25/23.75), and tetracycline $(T, 30)$. The interpretation of results was performed according to the CLSI guideline instructions [38-40].

\section{Conclusions}

Continuous monitoring of the prevalence and antimicrobial resistance profiles of Salmonella spp. on wild boars is useful for the epidemiological surveillance on territories, by means of animal sentinels. This kind of monitoring is also important for risk analysis on the consumption of meat and meat products of this species, which is traditionally consumed in our region as well as in many parts of Italy. In conclusion, the study underlines the possible role of wild boars in the diffusion of Salmonella spp. Moreover, due to the antimicrobial resistance found in our study, a risk in the spread of antimicrobial resistant strains can be supposed. Considering wild boars as sentinel, we can assert the low presence of antimicrobial resistance against "Highest Priority Critically Important" antimicrobials in the territory of the survey area.

Supplementary Materials: The following are available online at https://www.mdpi.com/article/10 .3390/pathogens10050568/s1, Table S1: Progressive number, lab. code, year of isolation, subtype or serotype, and antimicrobial susceptibility of isolated samples.

Author Contributions: Conceptualization, E.R., P.M., V.L., and C.E.; methodology, E.R., P.M., M.D., C.E., W.M., L.D., D.A., and M.P.; formal analysis, I.M., L.M., E.B., T.A., R.B., D.A., M.P., and L.D.; investigation, E.R., P.M., M.D., C.E., and W.M.; resources, E.R., C.E. and P.M.; data curation, V.L. and E.R.; writing—original draft preparation, E.R., P.M., and V.L.; writing—review and editing, W.M., M.D., L.S., C.E., L.D., M.P., D.M. and D.A.; supervision, C.E., E.R., and P.M.; project administration, C.E., E.R., W.M., P.M. and M.D.; funding acquisition, E.R., C.E. and P.M. All authors have read and agreed to the published version of the manuscript.

Funding: This research was funded by Italian Ministry of Health, grant number 12ALA and IZS PLV 08/17 RC, and the APC was funded by IZS PLV 08/17 RC.

Institutional Review Board Statement: Not applicable, nor humans or alive animals were involved in the study.

Informed Consent Statement: Not applicable.

Acknowledgments: The authors want to thank Walter Vencia, Fabrizio Lazzara, Valeria Cosma, and Valentina Franco for the skillful technical assistance; their work is gratefully acknowledged.

Conflicts of Interest: The authors declare no conflict of interest. 


\section{References}

1. European Food Safety Authority (EFSA); European Centre for Disease Prevention and Control (ECDC). The European Union One Health 2019 Zoonoses Report. EFSA J. 2021, 19, 6406.

2. Vieira-Pinto, M.; Morais, L.; Caleja, C.; Themudo, P.; Torres, C.; Igrejas, G.; Poeta, P.; Martins, C. Salmonella sp. in game (Sus scrofa and Oryctolagus cuniculus). Foodborne Pathog. Dis. 2011, 8, 739-740. [CrossRef]

3. Chiari, M.; Zanoni, M.; Tagliabue, S.; Lavazza, A.; Alborali, L.G. Salmonella serotypes in wild boars (Sus scrofa) hunted in northern Italy. Acta Vet. Scand. 2013, 55, 42. [CrossRef] [PubMed]

4. WHO. Antimicrobial Resistance: Global Report on Surveillance; World Health Organization: Geneva, Switzerland, 2014.

5. Torres, R.T.; Fernandes, J.; Carvalho, J.; Cunha, M.V.; Caetano, T.; Mendo, S.; Serrano, E.; Fonseca, C. Wild boar as a reservoir of antimicrobial resistance. Sci. Total Environ. 2020, 15, 717. [CrossRef] [PubMed]

6. Zottola, T.; Montagnaro, S.; Magnapera, C.; Sasso, S.; De Martino, L.; Bragagnolo, A.; D’Amici, L.; Condoleo, R.; Pisanelli, G.; Iovane, G.; et al. Prevalence and antimicrobial susceptibility of Salmonella in European wild boar (Sus scrofa); Latium Region-Italy. Comp. Immunol. Microbiol. Infect. Dis. 2013, 36, 161-168. [CrossRef]

7. Botti, V.; Navillod, F.V.; Domenis, L.; Orusa, R.; Pepe, E.; Robetto, S.; Guidetti, C. Salmonella spp. and antibiotic-resistant strains in wild mammals and birds in north-western Italy from 2002 to 2010. Vet. Ital. 2013, 49, 187-294.

8. Cilia, G.; Turchi, B.; Fratini, F.; Bilei, S.; Bossù, T.; De Marchis, M.L.; Cerri, D.; Pacini, M.I.; Bertelloni, F. Prevalence, Virulence and Antimicrobial Susceptibility of Salmonella spp.; Yersinia enterocolitica and Listeria monocytogenes in European Wild Boar (Sus scrofa) Hunted in Tuscany (Central Italy). Pathogens 2021, 10, 93. [CrossRef] [PubMed]

9. Navarro-Gonzalez, N.; Mentaberre, G.; Porrero, C.M.; Serrano, E.; Mateos, A.; López-Martín, J.M.; Lavín, S.; Domínguez, L. Effect of cattle on Salmonella carriage, diversity and antimicrobial resistance in free-ranging wild boar (Sus scrofa) in northeastern Spain. PLoS ONE 2012, 7, e51614. [CrossRef]

10. Massei, G.; Kindberg, J.; Licoppe, A.; Gačić, D.; Šprem, N.; Kamler, J.; Baubet, E.; Hohmann, U.; Monaco, A.; Ozolinšs, J.; et al. Wild boar populations up, numbers of hunters down? A review of trends and implications for Europe. Pest. Manag. Sci. 2015, 71, 492. [CrossRef] [PubMed]

11. Pittiglio, C.; Khomenko, S.; Beltran-Alcrudo, D. Wild boar mapping using population-density statistics: From polygons to high resolution raster maps. PLoS ONE 2018, 13, e0193295. [CrossRef] [PubMed]

12. Cilia, G.; Bertelloni, F.; Mignone, W.; Spina, S.; Berio, E.; Razzuoli, E.; Vencia, W.; Franco, V.; Cecchi, F.; Bogi, S.; et al. Molecular detection of Leptospira spp. in wild boar (Sus scrofa) hunted in Liguria region (Italy). Comp. Immunol. Microbiol. Infect. Dis. 2020, 68, 101410. [CrossRef] [PubMed]

13. Modesto, P.; De Ciucis, C.G.; Vencia, W.; Pugliano, M.C.; Mignone, W.; Berio, E.; Masotti, C.; Ercolini, C.; Serracca, L.; Andreoli, T.; et al. 2021 Evidence of antimicrobial resistance and presence of pathogenicity genes in Yersinia enterocolitica isolate from wild boars. Pathogens 2021, 10, 398. [CrossRef]

14. Decastelli, L.; Giaccone, V.; Mignone, W. Bacteriological examination of meat of wild boars shot down in Piedmont and Liguria, Italy. IBEX J. Mt. Ecol. 1995, 3, 88-89.

15. Paulsen, P.; Smulders, F.J.M.; Hilbert, F. Salmonella in meat from hunted game: A Central European perspective. Food Res. Int. 2012, 45, 609-616. [CrossRef]

16. Longo, A.; Losasso, C.; Vitulano, F.; Mastrorilli, E.; Turchetto, S.; Petrin, S.; Mantovani, C.; Dalla Pozza, M.C.; Ramon, E.; Conedera, G.; et al. Insight into an outbreak of Salmonella Choleraesuis var. Kunzendorf in wild boars. Vet. Microbiol. 2019, $238,108423$. [CrossRef]

17. Chirullo, B.; Pesciaroli, M.; Drumo, R.; Ruggeri, J.; Razzuoli, E.; Pistoia, C.; Petrucci, P.; Martinelli, N.; Cucco, L.; Moscati, L.; et al. Salmonella Typhimurium exploits inflammation to its own advantage in piglets. Front. Microbiol. 2015, 6, 985. [CrossRef]

18. Razzuoli, E.; Amadori, M.; Lazzara, F.; Bilato, D.; Ferraris, M.; Vito, G.; Ferrari, A. Salmonella serovar-specific interaction with jejunal epithelial cells. Vet. Microbiol. 2017, 207, 219-225. [CrossRef]

19. Wills, R.W.; Gray, J.T.; Fedorka-Cray, P.J.; Yoon, K.J.; Ladely, S.; Zimmerman, J.J. Synergism between porcine reproductive and respiratory syndrome virus (PRRSV) and Salmonella cholerasuis in swine. Vet. Microbiol. 2000, 71, 177-192. [CrossRef]

20. Razzuoli, E.; Mignone, G.; Lazzara, F.; Vencia, W.; Ferraris, M.; Masiello, L.; Vivaldi, B.; Ferrari, A.; Bozzetta, E.; Amadori, M. Impact of cadmium exposure on swine enterocytes. Toxicol. Lett. 2018, 287, 92-99. [CrossRef]

21. Gil Molino, M.; Risco Pérez, D.; Gonçalves Blanco, P.; Fernandez Llario, P.; Quesada Molina, A.; García Sánchez, A.; Cuesta Gerveno, J.M.; Gómez Gordo, L.; Martín Cano, F.E.; Pérez Martínez, R.; et al. Outbreaks of antimicrobial resistant Salmonella Choleraesuis in wild boars piglets from central-western Spain. Transbound. Emerg. Dis. 2019, 66, 225-233. [CrossRef]

22. Gil Molino, M.; García Sánchez, A.; Risco Pérez, D.; Gonçalves Blanco, P.; Quesada Molina, A.; Rey Pérez, J.; Martín Cano, F.E.; Cerrato Horrillo, R.; Hermoso-de-Mendoza Salcedo, J.; Fernández Llario, P. Prevalence of Salmonella spp. in tonsils, mandibular lymph nodes and faeces of wild boar from Spain and genetic relationship between isolates. Transbound. Emerg. Dis. 2019, 66, 1218-1226. [CrossRef]

23. Graziani, C.; Busani, L.; Dionisi, A.M.; Caprioli, A.; Ivarsson, S.; Hedenström, I.; Luzzi, I. Virulotyping of Salmonella enterica serovar Napoli strains isolated in Italy from human and nonhuman sources. Foodborne Pathog. Dis. 2011, 8, 997-1003. [CrossRef] [PubMed]

24. Sabbatucci, M.; Dionisi, A.M.; Pezzotti, P.; Lucarelli, C.; Barco, L.; Mancin, M.; Luzzi, I. Molecular and Epidemiologic Analysis of Re emergent Salmonella enterica Serovar Napoli, Italy, 2011-2015. Emerg. Infect. Dis. 2018, 24, 562-565. [CrossRef] [PubMed] 
25. ECDC European Centre for Disease Prevention and Control. Increase in Salmonella Stourbridge Infections in Germany during 2016; ECDC: Stockholm, Sweden, 2016.

26. ECDC European Centre for Disease Prevention and Control. Increase in Number of Salmonella Stourbridge Infections in Germany during 2016; Update; ECDC: Stockholm, Sweden, 2017.

27. Wacheck, S.; Fredriksson-Ahomaa, M.; König, M.; Stolle, A.; Stephan, R. Wild boars as an important reservoir for foodborne pathogens. Foodborne Pathog. Dis. 2010, 7, 307-312. [CrossRef]

28. Wilcock, B.P.; Schwartz, K. Salmonellosis. In Diseases in Swine, 7th ed.; Leman, A.D., Straw, B.E., Mengeling, W.E., D'Allaire, S., Taylor, D.J., Eds.; Iowa State University Press: Ames, IA, USA, 1992; pp. 570-583.

29. Gray, J.T.; Fedorka-Cray, P.J.; Stabel, T.S.; Kramer, T.T. Natural transmission of Salmonella choleraesuis in swine. Appl. Environ. Microbiol. 1996, 62, 141-146. [CrossRef]

30. Methner, U.; Heller, M.; Bocklisch, H. Salmonella enterica subspecies enterica serovar Choleraesuis in a wild boar population in Germany. Eur. J. Wildl. Res. 2009, 56, 493-502. [CrossRef]

31. Bonardi, S.; Bolzoni, L.; Zanoni, R.G.; Morganti, M.; Corradi, M.; Gilioli, S.; Pongolini, S. Limited Exchange of Salmonella among Domestic Pigs and Wild Boars in Italy. Ecohealth 2019, 16, 420-428. [CrossRef] [PubMed]

32. Radhouani, H.; Silva, N.; Poeta, P.; Torres, C.; Correia, S.; Igrejas, G. Potential impact of antimicrobial resistance in wildlife, environment and human health. Front. Microbiol. 2014, 5, 23. [CrossRef]

33. Sköld, O. Sulfonamide resistance: Mechanisms and trends. Drug Resist. Updates 2000, 3, 155-160. [CrossRef] [PubMed]

34. Grossman, T.H. Tetracycline Antibiotics and Resistance. Cold Spring Harb. Perspect. Med. 2016, 6, a025387. [CrossRef] [PubMed]

35. Caleja, C.; de Toro, M.; Gonçalves, A.; Themudo, P.; Vieira-Pinto, M.; Monteiro, D.; Rodrigues, J.; Sáenz, Y.; Carvalho, C.; Igrejas, G.; et al. Antimicrobial resistance and class I integrons in Salmonella enterica isolates from wild boars and Bísaro pigs. Int. Microbiol. 2011, 14, 19-24. [PubMed]

36. WHO. Available online: https:/ / www.who.int/foodsafety/publications/antimicrobials-sixth/en/ (accessed on 24 February 2021).

37. CLSI Document M02-A11. Performance Standards for Antimicrobial Disk Susceptibility Tests, 11th ed.; Clinical and Laboratory Standards Institute: Wayne, PA, USA, 2012.

38. CLSI Document M100-S17. Performance Standards for Antimicrobial Susceptibility Testing: Seventeenth Informational Supplement; Clinical and Laboratory Standards Institute: Wayne, PA, USA, 2007.

39. CLSI Document M100-S22. Performance Standards for Antimicrobial Susceptibility Testing: Twenty-Second Informational Supplement; Clinical and Laboratory Standards Institute: Wayne, PA, USA, 2012.

40. CLSI Document M100-S23. Performance Standards for Antimicrobial Susceptibility Testing: Twenty-Third Informational Supplement; Clinical and Laboratory Standards Institute: Wayne, PA, USA, 2014. 